\title{
Enzalutamide inhibits proliferation of gemcitabine-resistant bladder cancer cells with increased androgen receptor expression
}

\author{
KOJI KAMEYAMA ${ }^{1}$, KENGO HORIE $^{1}$, KOSUKE MIZUTANI $^{1}$, TAKU KATO ${ }^{1}$, YASUNORI FUJITA ${ }^{2}$, \\ KYOJIRO KAWAKAMI ${ }^{2}$, TOSHIO KOJIMA ${ }^{3}$, TATSUHIKO MIYAZAKI ${ }^{4}$, \\ TAKASHI DEGUCHI ${ }^{1}$ and MASAFUMI ITO ${ }^{2}$ \\ ${ }^{1}$ Department of Urology, Gifu University Graduate School of Medicine, Gifu, Gifu 501-1193; \\ ${ }^{2}$ Research Team for Mechanism of Aging, Tokyo Metropolitan Institute of Gerontology, Itabashi-ku, \\ Tokyo 173-0015; ${ }^{3}$ Health Support Center, Toyohashi University of Technology, Tenpaku-cho, Toyohashi, \\ Aichi 441-8580; ${ }^{4}$ Division of Pathology, Gifu University Hospital, Gifu, Gifu 501-1194, Japan
}

Received June 28, 2016; Accepted November 17, 2016

DOI: 10.3892/ijo.2016.3781

\begin{abstract}
Advanced bladder cancer is treated mainly with gemcitabine and cisplatin, but most patients eventually become resistance. Androgen receptor (AR) signaling has been implicated in bladder cancer as well as other types of cancer including prostate cancer. In this study, we investigated the expression and role of AR in gemcitabine-resistant bladder cancer cells and also the potential of enzalutamide, an AR inhibitor, as a therapeutic for the chemoresistance. First of all, we established gemcitabine-resistant T24 cells (T24GR) from T24 bladder cancer cells and performed gene expression profiling. Microarray analysis revealed upregulation of AR expression in T24GR cells compared with T24 cells. AR mRNA and protein expression was confirmed to be increased in T24GR cells, respectively, by quantitative RT-PCR and western blot analysis, which was associated with more potent AR transcriptional activity as measured by luciferase reporter assay. The copy number of AR gene in T24GR cells determined by PCR was twice as many as that of T24 cells. AR silencing by siRNA transfection resulted in inhibition of proliferation of T24GR cells. Cell culture in charcoal-stripped serum and treatment with enzalutamide inhibited growth of
\end{abstract}

Correspondence to: Dr Masafumi Ito, Research Team for Mechanism of Aging, Tokyo Metropolitan Institute of Gerontology, 35-2 Sakae-cho, Itabashi-ku, Tokyo 173-0015, Japan

E-mail: mito@tmig.or.jp

Dr Takashi Deguchi, Department of Urology, Gifu University Graduate School of Medicine, 1-1 Yanagido, Gifu, Gifu 501-1193, Japan

E-mail: deguchit@gifu-u.ac.jp

Abbreviations: AR, androgen receptor; T24GR, gemcitabine-resistant T24 cells; CSS, charcoal-stripped serum; CRPC, castration-resistant prostate cancer; FBS, fetal bovine serum; siRNA, small interfering RNA; DHT, dihydrotestosterone; CI, combination index

Key words: enzalutamide, gemcitabine-resistance, bladder cancer, androgen receptor
T24GR cells, which was accompanied by cell cycle arrest. AR transcriptional activity was found to be reduced in T24GR cells by enzalutamide treatment. Lastly, enzalutamide also inhibited cell proliferation of HTB5 bladder cancer cells that express AR and possess intrinsic resistance to gemcitabine. Our results suggest that enzalutamide may have the potential to treat patients with advanced gemcitabine-resistant bladder cancer with increased AR expression.

\section{Introduction}

Bladder cancer is one of the most commonly diagnosed cancers worldwide (1). The combination chemotherapy with methotrexate, vinblastine, doxorubicin, and cisplatin (M-VAC) was reported in 1985 (2) which thereafter became the standard chemotherapy for patients with bladder cancer (3). Nowadays, the combination chemotherapy of gemcitabine and cisplatin (GC) has replaced M-VAC therapy as a first-line treatment for bladder cancer patients, because GC exhibited similar efficacy to M-VAC with less adverse events $(4,5)$. Although bladder cancer is relatively sensitive to chemotherapy, drug-resistance is inevitable and occurs within a few years in most cases. However, there has been no clearly established second-line therapy for bladder cancer resistant to first-line chemotherapy (6).

Androgen receptor (AR), one of the steroid hormone receptors, is a transcriptional factor whose activity is modulated by androgens. AR plays an important role in the survival of prostate cancer cells (7). In addition, AR has been implicated in other types of cancer including bladder cancer as well as liver, kidney, breast, and lung cancer (8-11). A study demonstrated that downregulation of AR expression inhibited bladder cancer cell growth in vitro and in vivo (12). It has been also shown that expression of AR was higher in doxorubicin-resistant bladder cancer cells than sensitive cells and that androgen/AR signaling correlated with the effectiveness of doxorubicin (13).

In this study, in an attempt to identify novel therapeutic targets for gemcitabine-resistance, we developed gemcitabineresistant bladder cancer cells from sensitive cells and performed microarray analysis. We found that AR expression was upregulated in gemcitabine-resistant cancer cells compared with 
parental cells. We then investigated the effects on growth of gemcitabine-resistant cancer cells of AR knockdown as well as culture in charcoal-stripped serum (CSS) and treatment with enzalutamide, a second-generation non-steroidal AR inhibitor used for castration-resistant prostate cancer (CRPC) patients. We also studied the effect of enzalutamide on gemcitabineresistance. Finally, we examined if enzalutamide could inhibit growth of AR-expressing bladder cancer cells with intrinsic gemcitabine-resistance. Our results suggested that blockade of AR signaling by enzalutamide might be effective for patients with advanced gemcitabine-resistant bladder cancer with increased AR expression.

\section{Materials and methods}

Reagents and antibodies. Gemcitabine and enzalutamide were from Sigma-Aldrich(St.Louis,MO,USA) and MedChemExpress LLC (Princeton, NJ, USA), respectively. Dihydrotestosterone (DHT) was purchased from Tokyo Chemical Industry (Tokyo, Japan). Anti- $\beta$-actin (\#3700), anti-AR (\#5153), anti-cyclin D1 (\#2978), anti-cyclin B1 (\#12231), anti-caspase-3 (\#9662) and anti-cleaved caspase-3 (\#9664) antibodies were from Cell Signaling Technology (Danvers, MA, USA).

Cell culture and establishment of gemcitabine-resistant cells. T24 human bladder cancer cell line was obtained from RIKEN Cell Bank (Tsukuba, Japan). Cells were cultured in RPMI-1640 medium supplemented with $10 \%$ fetal bovine serum (FBS). T24 cells were initially cultured in the medium containing $5 \mathrm{nM}$ gemcitabine and the dose was gradually increased over 3 months. Finally, we obtained gemcitabineresistant T24 cells (T24GR) viable in the presence of $50 \mathrm{nM}$ gemcitabine. In specified experiments, cells were cultured in CSS from Thermo Fisher Scientific (Waltham, MA, USA). HTB5 human bladder cancer cell line was obtained from American Type Culture Collection (Manassas, VA, USA) and cultured in RPMI-1640 medium supplemented with 10\% FBS.

Cell growth and viability assay. The number of living cells was counted manually or automatically by using Countess Automated Cell Counter (Invitrogen, Carlsbad, CA, USA) after staining with trypan blue. The sensitivity to gemcitabine and enzalutamide was determined by WST-1 assay using the kit from Roche Diagnostics (Mannheim, Germany).

Microarray analysis of $m R N A$. Total RNA extracted by the miRNeasy Mini kit (Qiagen, Hilden, Germany) was used for the labeling and hybridization to the Agilent SurePrint G3 Human GE Microarray 8x60K (Agilent Technologies, Santa Clara, CA, USA). After washing, the microarrays were scanned on the Agilent DNA Microarray Scanner (Agilent Technologies). Raw data were collected using Agilent Feature Extraction software and analyzed using the GeneSpring GX11 (Agilent Technologies). The signal intensity of each probe was normalized by a percentile shift, in which each value was divided by the 75th percentile of all values in its array. Only the probes that had expression flags present under at least one condition were considered. Data were compared using t-tests, with a significance level set at $\mathrm{P}<0.05$. The remaining probes were selected using the criterion of at least a 3 -fold change.

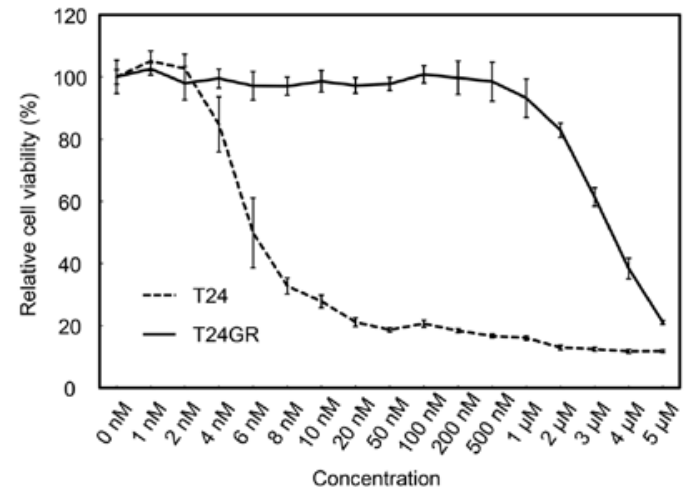

Figure 1. Viability of T24GR and T24 cells in the presence of gemcitabine. Cells $\left(3 \times 10^{3}\right)$ were seeded in a 96-well plate and cultured for $24 \mathrm{~h}$. Cells were then treated with gemcitabine at indicated concentrations for $72 \mathrm{~h}$ and subjected to WST assay. Data are expressed as mean \pm SD $(n=5)$.

The microarray data have been deposited in the NCBI Gene Expression Omnibus (GEO) and are accessible through the GEO Series accession no. GSE77883 (http://www.ncbi.nlm. nih.gov/geo/query/acc.cgi?acc=GSE77883).

Quantitative RT-PCR. Total RNA extracted using the miRNeasy Mini kit with on-column DNase I treatment was reverse transcribed using the ReverTra Ace qPCR RT Master Mix (Toyobo, Osaka, Japan). Quantitative RT-PCR was performed on the StepOnePlus Real-Time PCR system (Applied Biosystems, Foster City, CA, USA) using the Thunderbird SYBR qPCR Mix (Toyobo). PCR conditions include a denaturation at $95^{\circ} \mathrm{C} 1 \mathrm{~min}$, followed by 40 cycles of $15 \mathrm{sec}$ at $95^{\circ} \mathrm{C}$ and $1 \mathrm{~min}$ at $60^{\circ} \mathrm{C}$. Primer sequences were as follows: AR forward, 5'-TGTACACGTGGTCAAGTGGG-3'; reverse, 5'-GTGCATGCGGTACTCATTGAA-3'. 18S ribosomal RNA forward, 5'-GTAACCCGTTGAACCCCATT-3'; reverse, 5'-CCATCCAATCGGTAGTAGCG-3'. The relative expression was expressed as a ratio of AR to $18 \mathrm{~S}$ rRNA.

Western blot analysis. Whole cell lysates were prepared in lysis buffer [ $1 \%$ Nonidet P-40, $0.1 \%$ sodium deoxycholate, $0.1 \%$ sodium dodecyl sulfate, $1 \mathrm{mM}$ EDTA, $150 \mathrm{mM} \mathrm{NaCl}$, and $10 \mathrm{mM}$ Tris- $\mathrm{HCl}$ ( $\mathrm{pH}$ 7.4)] containing protease inhibitor cocktail (Sigma-Aldrich). Cell lysates were subjected to electrophoresis on $4-15 \%$ SDS-polyacrylamide gels (Bio-Rad, Hercules, CA, USA) and transferred to polyvinylidene difluoride membranes. After blocking in non-fat milk, membranes were incubated overnight at $4^{\circ} \mathrm{C}$ with primary antibodies followed by incubation for $60 \mathrm{~min}$ at room temperature with a horseradish peroxidaselinked secondary antibodies. Immunoreactive bands were detected by using the enhanced chemiluminescence (ECL) detection system (GE Healthcare).

Luciferase reporter assay. Twenty-four hours after seeding cells $\left(2.5 \times 10^{5}\right)$ into a 12-well plate, the pGL4 firefly luciferase reporter gene (Promega, Madison, WI, USA) containing 4 copies of the androgen response element (ARE; 5'-GCTAGC ACTTGCTGTTCTGCA-3') (14) upstream of the minimal promoter were co-transfected for $6 \mathrm{~h}$ with the Renilla expressing plasmid (phRL-TK, Promega) using Lipofectamine 2000 (Thermo Fisher Scientific). Firefly and Renilla luciferase 


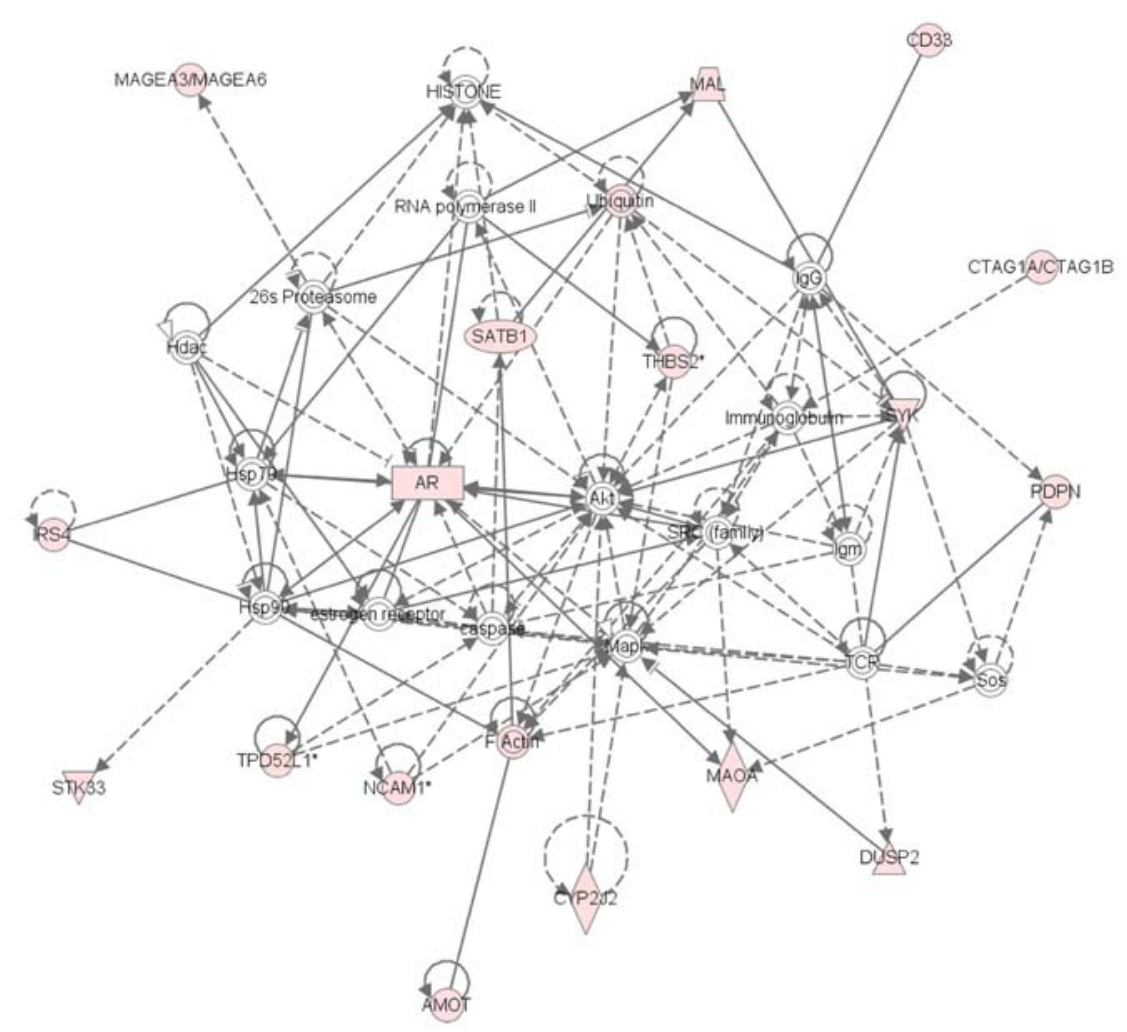

Figure 2. Network analysis of genes elevated in T24GR cells. Network analysis of 108 genes elevated by $>10$-fold in T24GR cells than in T24 cells revealed AR-related gene network (shown in pink) in T24GR cells.

activities were measured using the Pikkagene Dual Luciferase assay systems (Toyo B-Net, Tokyo, Japan). The AR transcriptional activity was expressed as a ratio of firefly to Renilla luciferase activity.

AR copy number assay. Genomic DNA was extracted using the NucleoSpin Tissue (Takara, Kusatsu, Japan). The copy number variation of AR gene was determined by using the TaqMan Copy Number assay (assay id: Hs00034522_cn, Applied Biosystems). The TaqMan Copy Number Reference assay (RNaseP, assay id: 4403326, Applied Biosystems) was used as an internal control. The assay was performed on the StepOnePlus PCR system using the TaqMan Genotyping Master Mix (Applied Biosystems) and $20 \mathrm{ng}$ of genomic DNA. PCR conditions were $95^{\circ} \mathrm{C}$ for $10 \mathrm{~min}$ for initial denaturation and enzyme activation, followed by 40 cycles of $15 \mathrm{sec}$ at $95^{\circ} \mathrm{C}$ and $1 \mathrm{~min}$ at $60^{\circ} \mathrm{C}$. The copy number of the AR gene was estimated from the $\mathrm{Ct}$ values by using the CopyCaller software (Applied Biosystems).

Small interfering RNA transfection. Two small interfering RNAs (siRNAs) for human AR gene were from Dharmacon GE Healthcare (Lafayette, CO, USA). Target sequences of the AR siRNAs were as follows: siRNA1, 5'-GGAACUCGAUC GUAUCAUU-3', siRNA2, 5'-UCAAGGAACUCGAUCG UAU-3'. As a negative control siRNA, siGENOME non-targeting siRNA control gene was used (Thermo Fisher Scientific). Each siRNA was transfected into cells for $6 \mathrm{~h}$ at $10 \mathrm{nM}$ using Lipofectamine 2000.

Evaluation of drug interaction. Drug interaction was assessed based on the method described by Chou and Talalay (15) and the combination index (CI) was determined using the CompuSyn software.

Statistical analysis. Student's t-test was used for comparison between two groups. Statistical significance was defined as a P-value of $<0.05$.

\section{Results}

Establishment of gemcitabine-resistant T24 bladder cancer cells. We developed gemcitabine-resistant T24 cells from parental T24 cells by treating cells with increasing concentrations of gemcitabine over 3 months. We performed WST-1 assay to examine the sensitivity of T24 and T24GR cells to gemcitabine. As shown in Fig. 1, T24GR cells were resistant to gemcitabine compared to parental $\mathrm{T} 24$ cells. The $\mathrm{IC}_{50}$ values of T2 4 cells and T24GR were $5.99 \mathrm{nM}$ and $3.46 \mu \mathrm{M}$, respectively (577.4-fold).

Increased expression of AR in T24GR cells. We investigated mRNA expression profiles of T24 and T24GR cells. Microarray analysis of mRNA expression showed that 596 genes were upregulated $>3$-fold in T24GR cells compared with T24 cells (data not shown). AR expression was 32-fold higher in T24GR cells than in T24 cells (Table I). Network analysis of 118 genes whose expression was elevated by $>10$-fold in T24GR cells than in T24 cells identified 5 gene networks, one of which was AR-related gene network (Fig. 2). As shown in Fig. 3A, quantitative RT-PCR confirmed that AR mRNA level in T24GR cells was significantly higher than that in T24 cells (40.5-fold). In accordance with the mRNA change, AR protein expression 
Table I. The genes with expression showing a >20-fold increase in T24GR cells compared with T24 cells.

\begin{tabular}{|c|c|c|}
\hline Gene symbol & Description & Fold chang \\
\hline LRRC38 & Homo sapiens leucine rich repeat containing 38 (LRRC38) & $5,918.9$ \\
\hline PASD1 & Homo sapiens PAS domain containing 1 (PASD1) & 849.3 \\
\hline FGF13 & Homo sapiens fibroblast growth factor 13 (FGF13), transcript variant 1 & 747.7 \\
\hline PDPN & Homo sapiens podoplanin (PDPN), transcript variant 2 & 499.8 \\
\hline ACTG2 & Homo sapiens actin, $\gamma 2$, smooth muscle, enteric (ACTG2), transcript variant 1 & 428.6 \\
\hline HS6ST2 & Homo sapiens heparan sulfate 6-O-sulfotransferase 2 (HS6ST2), transcript variant L & 259.3 \\
\hline GOLT1A & Homo sapiens golgi transport 1A (GOLT1A) & 106.2 \\
\hline CXorf57 & Homo sapiens chromosome $\mathrm{X}$ open reading frame 57 (CXorf57), transcript variant 1 & 104.5 \\
\hline CYP2J2 & Homo sapiens cytochrome P450, family 2, subfamily J, polypeptide 2 (CYP2J2) & 86.3 \\
\hline TSPAN18 & Homo sapiens tetraspanin 18 (TSPAN18) & 82.9 \\
\hline NELL2 & Homo sapiens NEL-like 2 (chicken) (NELL2), transcript variant 2 & 82.1 \\
\hline AMOT & Homo sapiens angiomotin (AMOT), transcript variant 2 & 74.8 \\
\hline KISS1 & Homo sapiens KiSS-1 metastasis-suppressor (KISS1) & 71.9 \\
\hline FAM155B & Homo sapiens family with sequence similarity 155 , member B (FAM155B) & 69.5 \\
\hline XK & Homo sapiens X-linked Kx blood group (McLeod syndrome) (XK) & 68.7 \\
\hline SYK & Homo sapiens spleen tyrosine kinase (SYK), transcript variant 1 & 61.7 \\
\hline XLOC_003505 & BROAD Institute lincRNA (XLOC_003505) & 61.0 \\
\hline BAGE & Homo sapiens B melanoma antigen (BAGE) & 59.2 \\
\hline STXBP6 & Homo sapiens syntaxin binding protein 6 (amisyn) (STXBP6) & 55.0 \\
\hline PGM5 & Homo sapiens phosphoglucomutase 5 (PGM5) & 52.9 \\
\hline MAGEB2 & Homo sapiens melanoma antigen family B, 2 (MAGEB2) & 51.9 \\
\hline NCAM1 & Homo sapiens neural cell adhesion molecule 1 (NCAM1), transcript variant 5 & 51.7 \\
\hline XLOC_010251 & BROAD Institute lincRNA (XLOC_010251) & 49.7 \\
\hline UBD & Homo sapiens ubiquitin D (UBD) & 49.2 \\
\hline JPH1 & Homo sapiens junctophilin 1 (JPH1) & 49.1 \\
\hline FAM230C & $\begin{array}{l}\text { PREDICTED: Homo sapiens family with sequence similarity } 230 \text {, member C (FAM230C), } \\
\text { transcript variant X1 }\end{array}$ & 48.9 \\
\hline LOC 100134317 & Homo sapiens uncharacterized LOC100134317 (LOC100134317) & 44.0 \\
\hline ITM2A & Homo sapiens integral membrane protein $2 \mathrm{~A}$ (ITM2A), transcript variant 1 & 40.3 \\
\hline XAGE2B & Homo sapiens $\mathrm{X}$ antigen family, member 2B (XAGE2B) & 40.1 \\
\hline GDA & Homo sapiens guanine deaminase (GDA), transcript variant 2 & 39.3 \\
\hline GPC4 & Homo sapiens glypican 4 (GPC4) & 38.9 \\
\hline SLITRK4 & Homo sapiens SLIT and NTRK-like family, member 4 (SLITRK4), transcript variant 2 & 37.5 \\
\hline FAM110C & Homo sapiens family with sequence similarity 110 , member C (FAM110C) & 35.6 \\
\hline IRS4 & PREDICTED: Homo sapiens insulin receptor substrate 4 (IRS4), transcript variant X1 & 35.4 \\
\hline FOXR2 & Homo sapiens forkhead box R2 (FOXR2) & 34.6 \\
\hline IGFBP5 & Homo sapiens insulin-like growth factor binding protein 5 (IGFBP5) & 34.1 \\
\hline AR & Homo sapiens androgen receptor (AR), transcript variant 1 & 32.2 \\
\hline CNN1 & Homo sapiens calponin 1 , basic, smooth muscle (CNN1) & 32.0 \\
\hline BMP2 & Homo sapiens bone morphogenetic protein 2 (BMP2) & 31.4 \\
\hline PCSK6 & Homo sapiens proprotein convertase subtilisin/kexin type 6 (PCSK6), transcript variant 3 & 31.1 \\
\hline WNT5A & $\begin{array}{l}\text { Homo sapiens wingless-type MMTV integration site family, member 5A (WNT5A), } \\
\text { transcript variant } 1\end{array}$ & 30.1 \\
\hline CECR7 & $\begin{array}{l}\text { Homo sapiens cat eye syndrome chromosome region, candidate } 7 \text { (non-protein coding) } \\
\text { (CECR7) }\end{array}$ & 29.3 \\
\hline PLCB4 & Homo sapiens phospholipase C, $\beta 4$ (PLCB4), transcript variant 2 & 29.1 \\
\hline IQCF1 & Homo sapiens IQ motif containing F1 (IQCF1) & 29.1 \\
\hline AWAT2 & Homo sapiens acyl-CoA wax alcohol acyltransferase 2 (AWAT2) & 29.1 \\
\hline XLOC_014512 & BROAD Institute lincRNA (XLOC_014512) & 26.9 \\
\hline
\end{tabular}


Table I. Continued.

Gene symbol

Description

Fold change

FTCDNL1 Homo sapiens formiminotransferase cyclodeaminase N-terminal like (FTCDNL1)

XLOC_005442 BROAD Institute lincRNA (XLOC_005442)

PLAC4

Homo sapiens placenta-specific 4 (PLAC4)

POTEB

Homo sapiens POTE ankyrin domain family, member B (POTEB)

GAL

Homo sapiens galanin/GMAP prepropeptide (GAL)

MAL

GABRG2

Homo sapiens mal, T-cell differentiation protein (MAL), transcript variant a

Homo sapiens $\gamma$-aminobutyric acid (GABA) A receptor, $\gamma 2$ (GABRG2), transcript variant 1

A

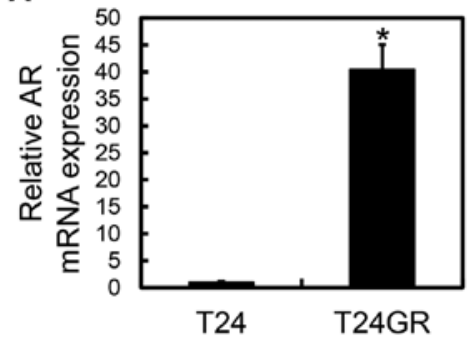

C

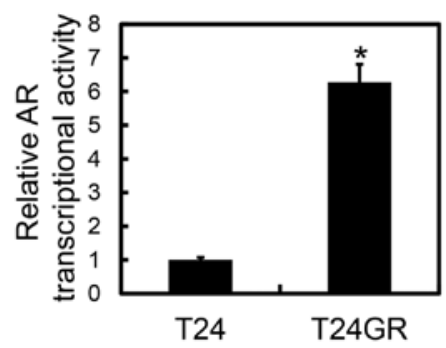

B
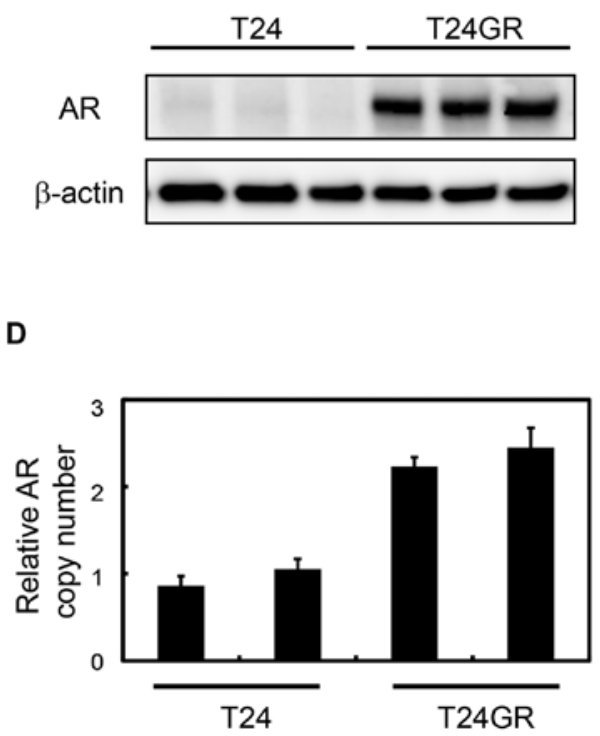

Figure 3. Transcription and expression of AR and copy number of the AR gene in T24 and T24GR cells. (A) Total RNA was isolated from T24 and T24GR cells and subjected to quantitative RT-PCR for AR and 18S rRNA. The relative expression was expressed as a ratio of AR to 18S rRNA. Data are expressed as mean \pm SD from three independent experiments in duplicate and asterisk indicates statistical significance compared with $\mathrm{T} 24$ cells $\left({ }^{*} \mathrm{P}<0.05\right)$. (B) Cell lysates were subjected to western blot analysis for AR and $\beta$-actin $(n=3)$. (C) The firefly luciferase reporter gene containing 4 copies of ARE and the Renilla luciferase expressing plasmid were transfected into cells $\left(2.5 \times 10^{5}\right)$ seeded in a 12-well plate. Six hours after transfection, the medium was replaced with normal medium. Twenty-four hours later, transfected T24 and T24GR cells were subjected to luciferase assay. The AR transcriptional activity was expressed as a ratio of firefly to Renilla luciferase activity. Data are expressed as mean \pm SD $(n=3)$ and asterisk indicates statistical significance compared with $\mathrm{T} 24$ cells $(* \mathrm{P}<0.05)$. (D) Genomic DNA was isolated from T24 and T24GR cells and the copy number of the AR gene was determined by using the TaqMan Copy Number assay. The copy number of the AR gene was estimated from the Ct values. Data are expressed as mean \pm SD $(n=4)$.

was markedly increased in T24GR cells compared with T24 cells (Fig. 3B). We also investigated the AR transcriptional activity in T24 and T24GR cells by luciferase reporter assays. Consistent with the increased expression of AR mRNA and protein, the AR transcriptional activity in T24 GR cells was about 6 times higher than that in T24 cells in normal medium (Fig. 3C). These results indicated that AR expression was increased in gemcitabine-resistant T24GR cells compared with gemcitabine-sensitive T24 cells, which was also shown to be functional. Since AR signaling has been implicated in bladder cancer (16), we selected and focused on AR in subsequent studies.

$A R$ gene copy number change contributed to increased $A R$ expression in $T 24 G R$ cells. In an attempt to identify the mechanism underlying the increased expression of AR in T24GR 
A

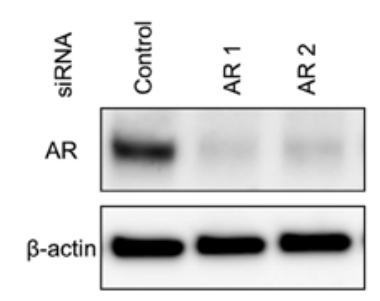

B

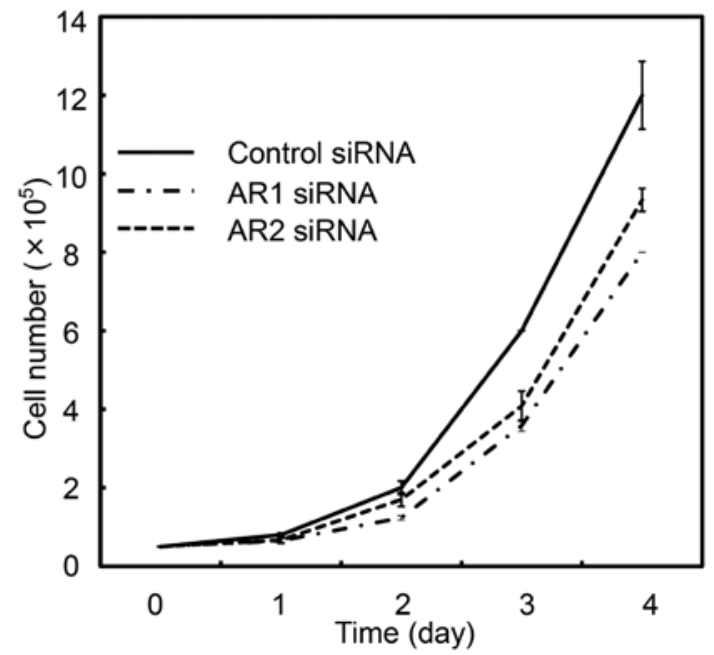

Figure 4. Effects of AR knockdown on proliferation of T24GR cells. T24GR cells were transfected with $10 \mathrm{nM}$ control, AR1 or AR2 siRNA and cultured for $48 \mathrm{~h}$. (A) Cell lysates were harvested and subjected to western blot analysis for AR and $\beta$-actin. (B) Twenty-four hours after siRNA transfection, 5x10 ${ }^{4}$ cells were seeded in a 6 -well plate and cultured in the medium containing FBS. Viable cell number was counted at indicated times after seeding by trypan blue dye exclusion assay. Data are expressed as mean $\pm \mathrm{SD}(\mathrm{n}=3)$.
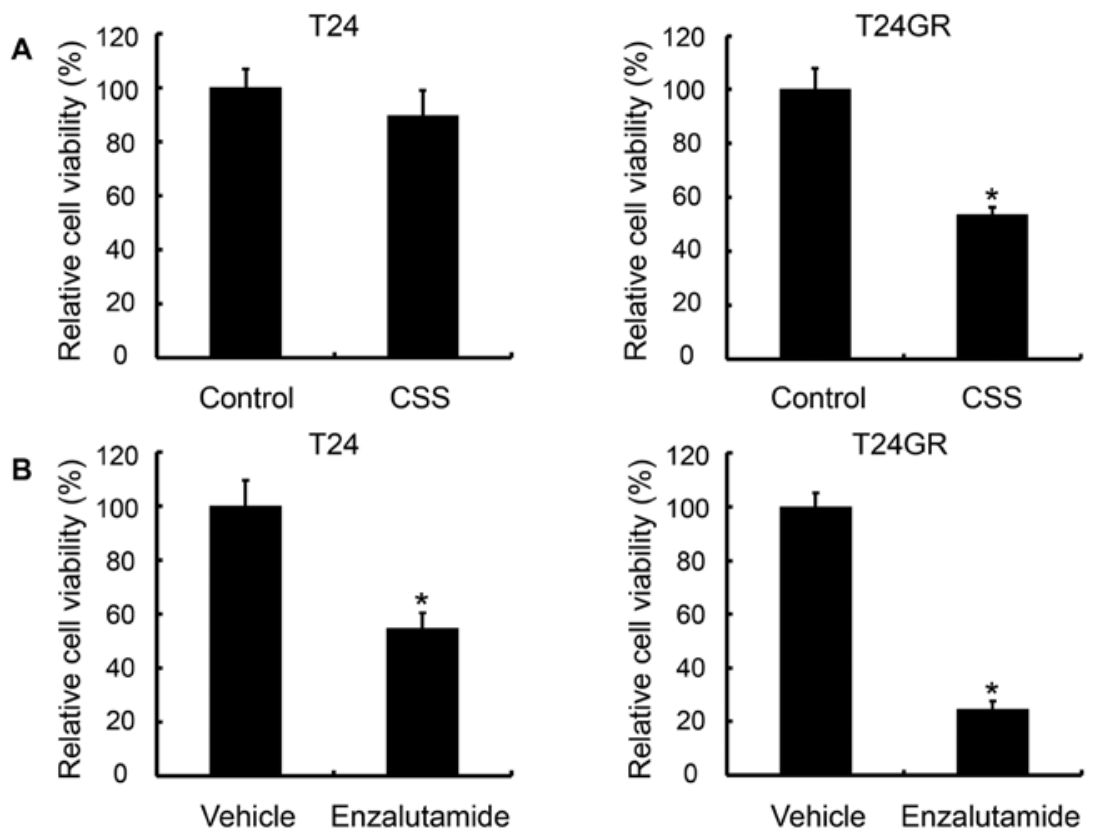

Figure 5. Effects of culture in CSS and treatment with enzalutamide on growth of T24GR cells. T24 and T24GR cells $\left(5 \times 10^{5}\right)$ were seeded in a 6-well plate and cultured for $24 \mathrm{~h}$. The culture medium was changed to that containing CSS or FBS (control) (A) or that containing $50 \mu \mathrm{M}$ enzalutamide or vehicle (DMSO) (B). Seventy-two hours later, viable cell number was counted by trypan blue dye exclusion assay. The cell number is presented relative to that of control or DMSO treatment (set as $100 \%)$. Data are expressed as mean $\pm \mathrm{SD}(\mathrm{n}=3)$ and asterisk indicates statistical significance compared with control (" $\mathrm{P}<0.05)$.

cells, we examined whether the AR gene copy number was changed between T24 and T24GR cells. The results showed that the number of AR gene copy in T24GR cells was twice as many as that of T24 cells (Fig. 3D), which was supposed to be one of the reasons for upregulation of AR in T24GR cells.

AR knockdown inhibited proliferation of T24GR cells. It has been reported that AR plays an important role in growth of bladder cancer cells $(12,13)$. Here we inhibited AR expression by siRNA transfection and examined the effect on prolifera- tion of T24GR cells cultured in the medium containing FBS. As shown in Fig. 4A, AR protein expression was effectively reduced by both AR1 siRNA and AR2 siRNA. The proliferation of AR siRNA-transfected cells was significantly inhibited compared to control siRNA-transfected cells (Fig. 4B). These results suggested that elevated AR expression was involved in proliferation of T24GR cells.

Culture in CSS and treatment with enzalutamide inhibited growth of T24GR cells. Since AR knockdown reduced growth 
A

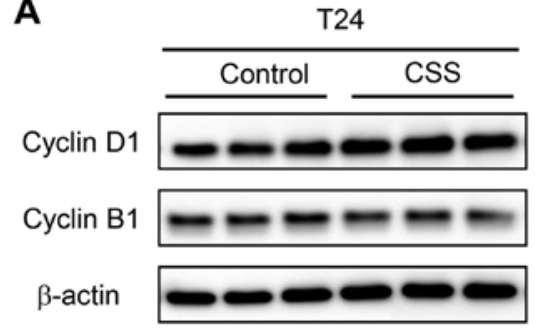

B

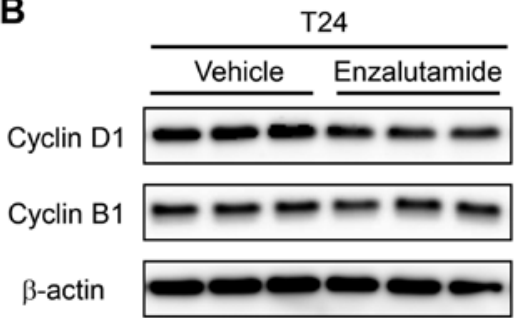

T24GR

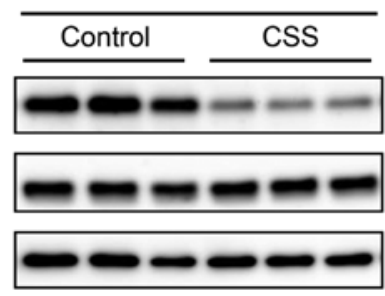

T24GR

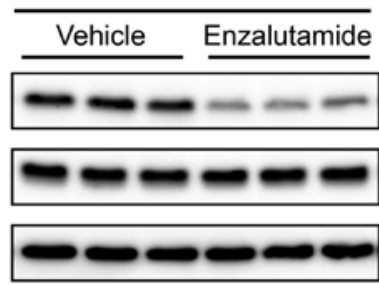

Figure 6. Effects of culture in CSS and treatment with enzalutamide on cell cycle of T24GR cells. T24 and T24GR cells (5x10 5 ) were seeded in a 6-well plate and cultured for $24 \mathrm{~h}$. The culture medium was changed to that containing CSS or FBS (control) (A) or that containing $50 \mu \mathrm{M}$ enzalutamide or vehicle (DMSO) (B). Seventy-two hours later, cell lysates were harvested and subjected to western blot analysis for cyclin B1, D1 and $\beta$-actin.

of T24GR cells, we examined the effect of androgen depletion and enzalutamide treatment. In order to deplete androgens, we cultured T24 and T24GR cells in the medium containing CSS or FBS and compared the cell viability. Culture in CSS did not affect the viability of T24 cells, but the number of viable T24GR cells cultured in CSS was lower than that cultured in FBS (Fig. 5A). However, the addition of DHT to the medium containing CSS did not promote growth of T24GR cells (data not shown). Next, we investigated the effects of enzalutamide, an AR inhibitor, on growth of both cell types. Enzalutamide inhibited the viability of T24GR cells significantly, whereas it also reduced that of T24 cells expressing little or no AR, although to a lesser extent (Fig. 5B). These results suggested that proliferation of T24GR cells depended on AR signaling and was inhibited by culture in CSS and treatment with enzalutamide. The absence of DHT effect on proliferation in CSS also suggested that AR might be activated other than androgens, which were present in FBS but not in CSS.

Growth inhibition of T24GR cells by culture in CSS and treatment with enzalutamide was mediated by cell cycle arrest. In order to investigate whether the inhibitory effects of culture in CSS and treatment with enzalutamide on the growth of T24GR cells are mediated through cell cycle arrest or apoptosis, we performed western blot analysis for cyclins and caspase-3. As shown in Fig. 6, expression of cyclin D1 was significantly lower in T24GR cells cultured in CSS and in those treated with enzalutamide. In T24 cells treated with enzalutamide, cyclin D1 expression was slightly decreased compared with those treated with vehicle, which was consistent with the enzalutamide effect on growth of T24 cells (Fig. 5B). Expression of cyclin B1 was not affected by culture in CSS and treatment with enzalutamide in either T24 or T24GR cells. On the other hand, cleaved caspase-3 was not detected in either T24 or T24GR cells under any experimental conditions tested, although caspase- 3 was expressed at the same level (data not shown). These results suggested that culture in CSS and treatment with enzalutamide might inhibit

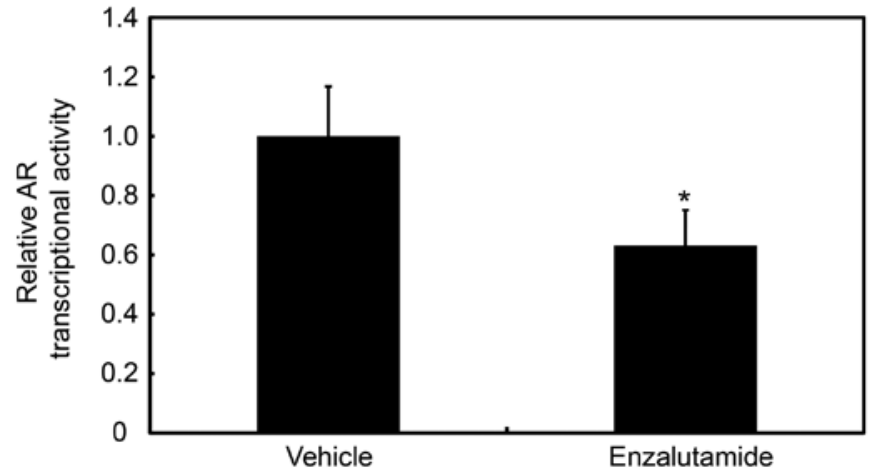

Figure 7. Effects of enzalutamide on AR transcriptional activity in T24GR cells. The firefly luciferase reporter gene containing 4 copies of ARE and the Renilla luciferase expressing plasmid were transfected into cells $\left(2.5 \times 10^{5}\right)$ seeded in a 12-well plate. Six hours after transfection, the medium was replaced with normal medium. After $24 \mathrm{~h}$ culture in normal medium, cells were treated with $50 \mu \mathrm{M}$ enzalutamide or vehicle (DMSO) for $24 \mathrm{~h}$ prior to luciferase assay. The AR transcriptional activity was expressed as a ratio of firefly to Renilla luciferase activity. Data are expressed as mean $\pm \mathrm{SD}(\mathrm{n}=3)$ and asterisk indicates statistical significance compared with vehicle ( $(\mathrm{P}<0.05)$.

growth of T24GR cells via induction of cell cycle arrest but not of apoptosis.

Inhibition of AR transcriptional activity by enzalutamide treatment in T24GR cells. Enzalutamide exerts an antitumor activity through preventing androgen binding to the ligand-binding domain of AR, and also through inhibiting AR nuclear translocation, DNA binding, and coactivator recruitment $(17,18)$. Here we measured the AR transcriptional activity in T24GR cells treated with enzalutamide. The AR transcriptional activity was decreased in T24GR cells treated with enzalutamide compared with those treated with vehicle (Fig. 7).

Effects of enzalutamide on gemcitabine-resistance in T24GR cells. In order to study the combined effects, we treated 


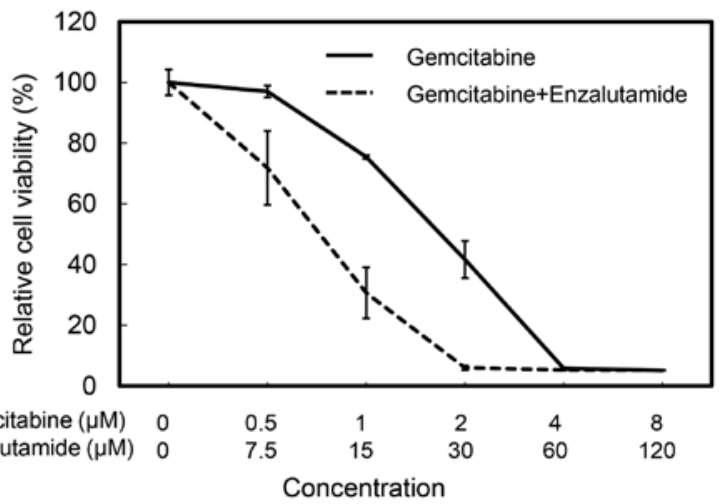

B

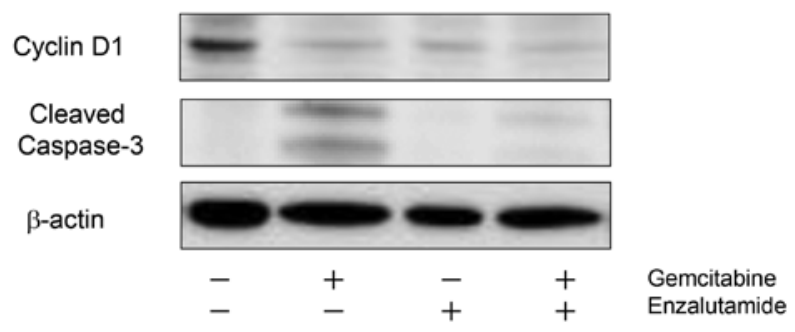

Figure 8. Effects of enzalutamide on gemcitabine-resistance in T24GR cells. (A) Cells $\left(1 \times 10^{3}\right)$ seeded in a 96-well plate were treated with enzalutamide and gemcitabine for $120 \mathrm{~h}$. Cells were then subjected to WST assay. Data are expressed as mean $\pm \mathrm{SD}(\mathrm{n}=3)$. (B) Cells $\left(2 \times 10^{4}\right)$ seeded in $35-\mathrm{mm}$ dish were treated with enzalutamide $(60 \mu \mathrm{M})$ and/or gemcitabine $(2 \mu \mathrm{M})$ for $72 \mathrm{~h}$. Cell lysates were then harvested and subjected to western blot analysis for cyclin D1, cleaved caspase- 3 and $\beta$-actin.

cells with enzalutamide and gemcitabine. Treatment with enzalutamide reduced viability of T24GR cells treated with gemcitabine (Fig. 8A). The calculated CI was 0.847 at $\mathrm{Fa}$ of 0.5 , showing synergy between these two drugs. Western blot analysis demonstrated that gemcitabine, enzalutamide and both decreased cyclin D1 expression. However, the level of cleaved caspase-3 was decreased by the co-presence of enzalutamide (Fig. 8B), indicating that enzalutamide did not enhance but rather diminished cleavage of caspase- 3 mediated by gemcitabine. These results suggested that enzalutamide could attenuate gemcitabine-resistance of T24GR cells.

Growth inhibition of HTB5 cells by enzalutamide. Lastly, we investigated the effects of enzalutamide in HTB5 bladder cancer cell line that was shown to express AR and possess intrinsic resistance to gemcitabine $(19,20)$. Treatment with enzalutamide inhibited cell proliferation (Fig. 9A), which was accompanied by a decrease in cyclin D1 expression (Fig. 9B). These results suggest that enzalutamide inhibits growth of AR-expressing bladder cancer cells with intrinsic (HTB5) as well as acquired (T24GR) resistance to gemcitabine.

\section{Discussion}

Gemcitabine, difluorodeoxycytidine, is widely used for treatment of many types of cancer including lung, pancreatic, biliary tract, breast, and urothelial cancer, but most patients treated with gemcitabine eventually acquire resistance. Although genes involved in drug transport and those involved in drug metabolism have been proposed to be involved in gemcitabine resistance (21-25) the underlying mechanisms remain still unclear. In an attempt to identify novel therapeutic targets for gemcitabine-resistance, we developed gemcitabine-resistant bladder cancer cells from sensitive cells and performed microarray analysis. We found that expression of functional AR was elevated in T24GR cells compared with T24 cells, which was shown to be caused in part due to increased AR gene copy number.

AR plays an important role in the survival of prostate cancer cells (7). Androgen deprivation therapy therefore has been used as a primary treatment for patients with prostate cancer, but eventually becomes ineffective, the condition of which is designated as CRPC. AR mutation and amplification as well as deregulation of AR co-regulator/activator have been proposed to be responsible for castration-resistance $(26,27)$. It has been also demonstrated that AR splice variants influence sensitivity to taxane, which is used for the treatment of CRPC (28). On the other hand, AR has been implicated
A

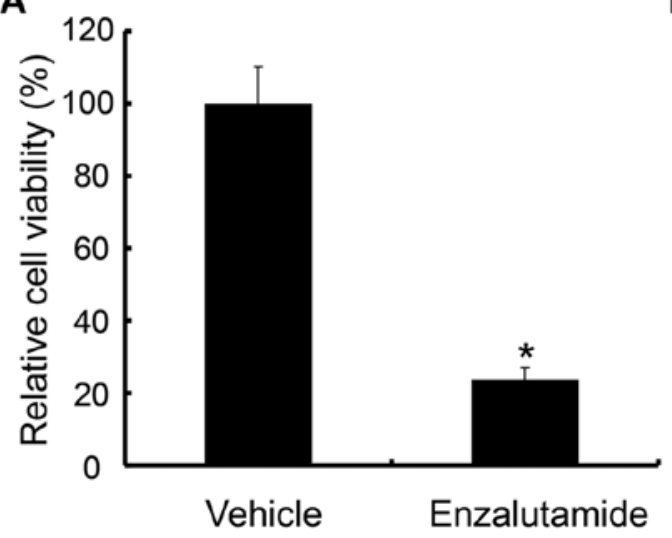

B

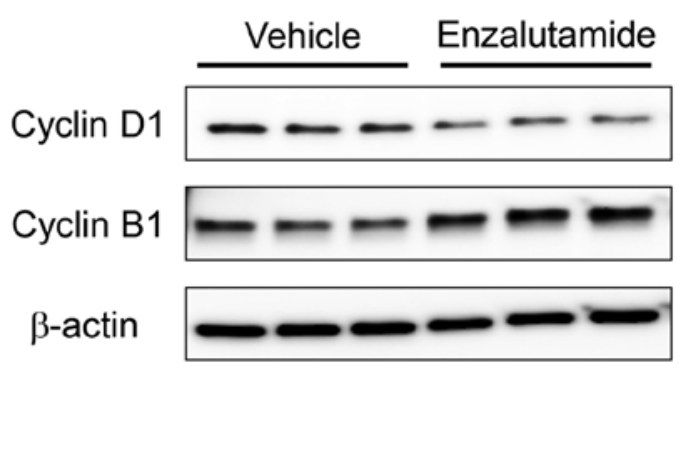

Figure 9. Effects of enzalutamide on proliferation in HTB5 cells. (A) Cells $\left(5 \times 10^{4}\right)$ seeded in a 6 -well plate were cultured for 24 h. The culture medium was changed to that containing $120 \mu \mathrm{M}$ enzalutamide or vehicle (DMSO) and cultured for $72 \mathrm{~h}$. Viable cell number was counted by trypan blue dye exclusion assay. The cell number is presented relative to that of DMSO treatment (set as $100 \%)$. Data are expressed as mean \pm SD ( $=3$ ) and asterisk indicates statistical significance compared with control ( $\mathrm{P}<0.05)$. (B) Cells $\left(1 \times 10^{5}\right)$ seeded in 6 -well plate were treated with enzalutamide $(120 \mu \mathrm{M})$ for $72 \mathrm{~h}$. Cell lysates were then harvested and subjected to western blot analysis for cyclin D1, cyclin B1 and $\beta$-actin. 
in a variety of cancers including bladder cancer (8-11) and also in drug-resistance cancers such as doxorubicin-resistant bladder cancer (13) as well as cisplatin-resistant endometrial cancer and taxol-resistant ovarian cancer $(29,30)$. As far as we know, our study is the first report that associated AR with gemcitabine-resistance of cancer cells.

Since AR signaling has been implicated in cancer, it would be possible that the high level of AR might favor growth and survival of T24GR cells. Indeed, AR knockdown reduced growth of gemcitabine-resistant T24GR cells. In addition, culture in the medium containing CSS inhibited growth of T24GR cells overexpressing AR, which was found to be mediated in part by induction of cell cycle arrest. However, DHT did not promote proliferation of T24GR cells cultured in the medium containing CSS. CSS is depleted not only of steroid hormones but also of some growth factors, cytokines and nutrients. The lack of proliferation by DHT might be because AR signaling was activated by other factors than androgens, which were present in FBS but absent in CSS. Growth inhibition in the medium containing CSS may be ascribed to the depletion of some important factors for the survival of T24GR cells.

These results led us to hypothesize that anti-androgen drugs could be a new treatment for chemoresistant bladder cancer. Enzalutamide is a second-generation non-steroidal AR inhibitor used for CRPC patients, which has a greater affinity for AR than conventional anti-androgen agents such as bicalutamide (18). It has been also shown that the underlying mechanisms of enzalutamide action are distinct from other anti-androgen drugs in that enzalutamide inhibits not only androgen binding to the ligand-binding domain of AR but also AR nuclear translocation, DNA binding and coactivator recruitment (17). We demonstrated that enzalutamide inhibited growth of T24GR cells more potently than T24 cells through inducing cell cycle arrest. This is consistent with the finding that AR expression was much higher in T24GR cells than in T24 cells. However, enzalutamide also inhibited growth of T24 cells expressing little or no AR, although to a lesser extent, which was also associated with cell cycle arrest. These results suggest the presence of AR-independent mechanisms of enzalutamide to inhibit cell growth. Alternatively, a residual amount of AR in T24 cells might take part in the inhibition. The underlying mechanism remains to be clarified by future studies. Lastly, we showed that the AR transcriptional activity was reduced by enzalutamide treatment in T24GR cells cultured in normal medium. Taken together, it was suggested that enzalutamide inhibits growth of AR-positive T24GR cells through suppressing AR signaling which is presumably activated by non-androgen ligands and also of AR-negative T24 cells through AR-independent mechanism.

A recent report revealed that a risk for bladder cancer recurrence was significantly lower in patients having received androgen deprivation therapy for prostate cancer (31), suggesting a role of AR signaling for cancer initiation, promotion and progression. Mashhadi et al evaluated 120 bladder cancer patients and examined relations between AR expression and tumor stage or grade (32). The patients with AR-positive tumor showed worse prognosis than those with AR-negative tumor. However, there are some conflicting reports showing that loss of AR was associated with high-grade and high-stage bladder cancer (33). Thus, the significance of AR in bladder cancer appears to be controversial in pathological and clinical studies. It remains to be studied whether AR is upregulated in patients with gemcitabine-resistant bladder cancer and whether AR expression correlates with gemcitabine-efficacy.

In this study, we showed that enzalutamide inhibits growth of AR-expressing bladder cancer cells with both acquired and intrinsic gemcitabine-resistance through cell cycle arrest. We also found that enzalutamide could attenuate gemcitabineresistance of T24GR cells. However, enzalutamide that causes a cytostatic response did not enhance but rather diminished an apoptotic response mediated by gemcitabine. The precise mechanisms underlying the combined effects remain to be studied.

It has been very recently reported that AR expression is elevated in cisplatin-resistant bladder cancer cells and is associated with resistance to cisplatin-based neoadjuvant chemotherapy (34). Furthermore, AR inactivation sensitized bladder cancer cells to cisplatin treatment. The authors also showed that enzalutamide inhibits the growth and AR expression/transcriptional activity in bladder cancer cells (35). Taken together, our study and others provided evidence that AR could be a therapeutic target for bladder cancer patients who exhibited resistance to GC therapy.

In conclusion, we demonstrated that enzalutamide inhibits proliferation of AR-expressing bladder cancer cell lines with acquired and intrinsic gemcitabine-resistance, suggesting that enzalutamide might be effective for patients with advanced gemcitabine-resistant bladder cancer with increased AR expression. The efficacy of enzalutamide treatment in patients with advanced bladder cancer needs to be established by clinical studies.

\section{Acknowledgements}

This study was supported in part by the Grant-in-Aid from The Ministry of Education, Culture, Sports, Science, and Technology of Japan (C-25861416 to T.K.).

\section{References}

1. Torre LA, Bray F, Siegel RL, Ferlay J, Lortet-Tieulent J and Jemal A: Global cancer statistics, 2012. CA Cancer J Clin 65: 87-108, 2015.

2. Sternberg CN, Yagoda A, Scher HI, Watson RC, Ahmed T, Weiselberg LR, Geller N, Hollander PS, Herr HW, Sogani PC, et al: Preliminary results of M-VAC (methotrexate, vinblastine, doxorubicin and cisplatin) for transitional cell carcinoma of the urothelium. J Urol 133: 403-407, 1985.

3. Loehrer PJ Sr, Einhorn LH, Elson PJ, Crawford ED, Kuebler P, Tannock I, Raghavan D, Stuart-Harris R, Sarosdy MF, Lowe BA, et al: A randomized comparison of cisplatin alone or in combination with methotrexate, vinblastine, and doxorubicin in patients with metastatic urothelial carcinoma: A cooperative group study. J Clin Oncol 10: 1066-1073, 1992.

4. von der Maase H, Hansen SW, Roberts JT, Dogliotti L, Oliver T, Moore MJ, Bodrogi I, Albers P, Knuth A, Lippert CM, et al: Gemcitabine and cisplatin versus methotrexate, vinblastine, doxorubicin, and cisplatin in advanced or metastatic bladder cancer: Results of a large, randomized, multinational, multicenter, phase III study. J Clin Oncol 18: 3068-3077, 2000.

5. von der Maase H, Sengelov L, Roberts JT, Ricci S, Dogliotti L, Oliver T, Moore MJ, Zimmermann A and Arning M: Long-term survival results of a randomized trial comparing gemcitabine plus cisplatin, with methotrexate, vinblastine, doxorubicin, plus cisplatin in patients with bladder cancer. J Clin Oncol 23: 4602-4608, 2005. 
6. Yafi FA, North S and Kassouf W: First- and second-line therapy for metastatic urothelial carcinoma of the bladder. Curr Oncol 18: e25-e34, 2011

7. Feldman BJ and Feldman D: The development of androgenindependent prostate cancer. Nat Rev Cancer 1: 34-45, 2001

8. Chia K, O'Brien M, Brown M and Lim E: Targeting the androgen receptor in breast cancer. Curr Oncol Rep 17: 4, 2015.

9. Ha YS, Lee GT, Modi P, Kwon YS, Ahn H, Kim WJ and Kim IY: Increased expression of androgen receptor mRNA in human renal cell carcinoma cells is associated with poor prognosis in patients with localized renal cell carcinoma. J Urol 194: 1441-1448, 2015

10. Ma WL, Lai HC, Yeh S, Cai X and Chang C: Androgen receptor roles in hepatocellular carcinoma, fatty liver, cirrhosis and hepatitis. Endocr Relat Cancer 21: R165-R182, 2014.

11. Mikkonen L, Pihlajamaa P, Sahu B, Zhang FP and Jänne OA Androgen receptor and androgen-dependent gene expression in lung. Mol Cell Endocrinol 317: 14-24, 2010.

12. Wu JT, Han BM, Yu SQ, Wang HP and Xia SJ: Androgen receptor is a potential therapeutic target for bladder cancer. Urology 75 820-827, 2010.

13. Shiota M, Takeuchi A, Yokomizo A, Kashiwagi E, Tatsugami K, Kuroiwa $\mathrm{K}$ and Naito S: Androgen receptor signaling regulates cell growth and vulnerability to doxorubicin in bladder cancer J Urol 188: 276-286, 2012.

14. Siddique HR, Mishra SK, Karnes RJ and Saleem M: Lupeol, a novel androgen receptor inhibitor: Implications in prostate cancer therapy. Clin Cancer Res 17: 5379-5391, 2011.

15. Chou TC and Talalay P: Quantitative analysis of dose-effect relationships: The combined effects of multiple drugs or enzyme inhibitors. Adv Enzyme Regul 22: 27-55, 1984.

16. Li Y, Izumi K and Miyamoto H: The role of the androgen receptor in the development and progression of bladder cancer. Jpn J Clin Oncol 42: 569-577, 2012

17. Chen Y, Clegg NJ and Scher HI: Anti-androgens and androgendepleting therapies in prostate cancer: New agents for an established target. Lancet Oncol 10: 981-991, 2009.

18. Tran C, Ouk S, Clegg NJ, Chen Y, Watson PA, Arora V, Wongvipat J, Smith-Jones PM, Yoo D, Kwon A, et al: Development of a second-generation antiandrogen for treatment of advanced prostate cancer. Science 324: 787-790, 2009.

19. Jeon HG, Yoon CY, Yu JH, Park MJ, Lee JE, Jeong SJ, Hong SK, Byun SS and Lee SE: Induction of caspase mediated apoptosis and down-regulation of nuclear factor- $\mathrm{KB}$ and Akt signaling are involved in the synergistic antitumor effect of gemcitabine and the histone deacetylase inhibitor trichostatin A in human bladder cancer cells. J Urol 186: 2084-2093, 2011.

20. Miyamoto H, Yang Z, Chen YT, Ishiguro H, Uemura H, Kubota Y, Nagashima Y, Chang YJ, Hu YC, Tsai MY, et al: Promotion of bladder cancer development and progression by androgen receptor signals. J Natl Cancer Inst 99: 558-568, 2007.

21. Duxbury MS, Ito H, Zinner MJ, Ashley SW and Whang EE: RNA interference targeting the M2 subunit of ribonucleotide reductase enhances pancreatic adenocarcinoma chemosensitivity to gemcitabine. Oncogene 23: 1539-1548, 2004.

22. Giovannetti E, Del Tacca M, Mey V, Funel N, Nannizzi S, Ricci S, Orlandini C, Boggi U, Campani D, Del Chiaro M, et al: Transcription analysis of human equilibrative nucleoside transporter-1 predicts survival in pancreas cancer patients treated with gemcitabine. Cancer Res 66: 3928-3935, 2006.
23. Nakahira S, Nakamori S, Tsujie M, Takahashi Y, Okami J, Yoshioka S, Yamasaki M, Marubashi S, Takemasa I, Miyamoto A, et al: Involvement of ribonucleotide reductase M1 subunit overexpression in gemcitabine resistance of human pancreatic cancer. Int J Cancer 120: 1355-1363, 2007.

24. Ohhashi S, Ohuchida K, Mizumoto K, Fujita H, Egami T, Yu J, Toma H, Sadatomi S, Nagai E and Tanaka M: Downregulation of deoxycytidine kinase enhances acquired resistance to gemcitabine in pancreatic cancer. Anticancer Res 28B 2205-2212, 2008.

25. Skrypek N, Duchêne B, Hebbar M, Leteurtre E, van Seuningen I and Jonckheere N: The MUC4 mucin mediates gemcitabine resistance of human pancreatic cancer cells via the concentrative nucleoside transporter family. Oncogene 32: 1714-1723, 2013.

26. Harris WP, Mostaghel EA, Nelson PS and Montgomery B: Androgen deprivation therapy: Progress in understanding mechanisms of resistance and optimizing androgen depletion. Nat Clin Pract Urol 6: 76-85, 2009.

27. Tombal B: What is the pathophysiology of a hormone-resistant prostate tumour? Eur J Cancer 47 (Suppl 3): S179-S188, 2011.

28. Thadani-Mulero M, Portella L, Sun S, Sung M, Matov A, Vessella RL, Corey E, Nanus DM, Plymate SR and Giannakakou P: Androgen receptor splice variants determine taxane sensitivity in prostate cancer. Cancer Res 74: 2270-2282, 2014.

29. Chen L, Chang WC, Hung YC, Chang YY, Bao BY, Huang HC, Chung WM, Shyr CR and Ma WL: Androgen receptor increases CD133 expression and progenitor-like population that associate with cisplatin resistance in endometrial cancer cell line. Reprod Sci 21: 386-394, 2014.

30. Sun NK, Huang SL, Lu HP, Chang TC and Chao CC: Integrative transcriptomics-based identification of cryptic drivers of taxolresistance genes in ovarian carcinoma cells: Analysis of the androgen receptor. Oncotarget 6: 27065-27082, 2015.

31. Izumi K, Taguri M, Miyamoto H, Hara Y, Kishida T, Chiba K, Murai T, Hirai K, Suzuki K, Fujinami K, et al: Androgen deprivation therapy prevents bladder cancer recurrence. Oncotarget 5: 12665-12674, 2014.

32. Mashhadi R, Pourmand G, Kosari F, Mehrsai A, Salem S, Pourmand MR, Alatab S, Khonsari M, Heydari F, Beladi L, et al: Role of steroid hormone receptors in formation and progression of bladder carcinoma: A case-control study. Urol J 11: 1968-1973, 2014.

33. Miyamoto H, Yao JL, Chaux A, Zheng Y, Hsu I, Izumi K, Chang C, Messing EM, Netto GJ and Yeh S: Expression of androgen and oestrogen receptors and its prognostic significance in urothelial neoplasm of the urinary bladder. BJU Int 109: 1716-1726, 2012.

34. Kashiwagi E, Ide H, Inoue S, Kawahara T, Zheng Y, Reis LO, Baras AS and Miyamoto H: Androgen receptor activity modulates responses to cisplatin treatment in bladder cancer. Oncotarget: Jun 14, 2016 (Epub ahead of print). doi: 10.18632/oncotarget.9994.

35. Kawahara T, Ide H, Kashiwagi E, El-Shishtawy KA, Li Y, Reis LO, Zheng Y and Miyamoto H: Enzalutamide inhibits androgen receptor-positive bladder cancer cell growth. Urol Oncol 34: 432.e15-23, 2016 